\title{
La puntura ad occhiello: l'esperienza dei Centri Dialisi della Azienda USL di Rimini
}

\author{
Marina Sgreccia' ${ }^{1}$, Manuela Molari², Deborah Pacifero², Sabrina Cecchini ${ }^{3}$, Stella Fabbrucci ${ }^{4}$, \\ Patrizia Fronda ${ }^{4}$, Marisa Tamagnini ${ }^{4}$, Patrizia Bignardi ${ }^{2}$
}

${ }^{1}$ Coordinatore Infermieristico Dialisi Aziendali, Azienda USL Rimini, Rimini

${ }^{2}$ Infermiere Dialisi, Ospedale Infermi, Rimini

${ }^{3}$ Infermiere CAL, Ospedale Ceccarini, Riccione (RN)

${ }^{4}$ Infermiere CAL, Ospedale Franchini, Santarcangelo di Romagna (RN)

\begin{abstract}
Five YeARS OF BUTTONHOLE TECHNIQUE IN THE DIALYSIS CENTERS OF THE LOCAL HEALTH DiSTRICT OF RIMINI
Abstract. The buttonhole technique represents an arteriovenous fistula puncture tool that allows positioning the needle always at the same site. This procedure not only is more comfortable for patients, but is also appreciated by nurses because it reduces hemostasis time and offers the advantage of an easy cannulation.

The only negative outcome of the buttonhole technique is, possibly, an increased incidence of skin infections. We introduced the buttonhole technique at the dialysis centers of the Rimini Hospital in 2009 and we here investigate the results and complications recorded during the following 5 years.
\end{abstract}

Key words: Buttonhole tecnique, Reducing pain, Easy cannulation, Skin infection

Conflict of interest: None.

Financial support: None.

Accettato: 27 Febbraio 2014

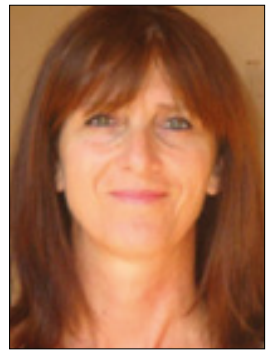

Marina Sgreccia

\section{Introduzione}

Le persone in trattamento emodialitico oggi hanno un'età biologica media elevata e un maggior numero di comorbidità (vasculopatie, diabete, ecc.), che rendono il confezionamento chirurgico della fistola arterovenosa (FAV) e il suo mantenimento parametri di elevata criticità assistenziale che necessitano di un approccio gestionale competente e strutturato (1).

L'utilizzo di un accesso vascolare ben funzionante è indispensabile per effettuare una dialisi adeguata. La gestione degli accessi vascolari in emodialisi (FAV, protesi e cateteri venosi centrali) è una competenza infermieristica ormai diffusa e confermata da studi nazionali e internazionali (2).

Il Dialysis Outcomes and Practice Patterns Study (DOPPS) ha dimostrato che la fistola arterovenosa (FAV) con vasi nativi è il tipo di accesso vascolare privilegiato e che, per questo, dovrebbe essere l'opzione di prima scelta per tutti i pazienti che necessitano di emodialisi (3).

L'errore più frequente nell'uso della FAV risiede nella tendenza a praticare la venipuntura reiterata nella stessa sede. Concorrono ad alimentare questa malpractice, estremamente diffusa, gli atteggiamenti sia dell'operatore che del paziente. L'operatore preferisce infiggere gli aghi sempre nella stessa sede, perché è più rapido, più facile e non si corre il rischio di insuccessi.

Il paziente richiede la stessa cosa, in quanto la sede iperutilizzata si desensibilizza progressivamente sino a rendere (in certi casi) del tutto indolore la venipuntura (4).

La tecnica di puntura a scala di corda prevede la rotazione del sito. Ad ogni seduta dialitica si punge in una sede alcuni $\mathrm{mm}$ al di sopra o al di sotto di quella precedente. Le cicatrici conseguenti si distribuiscono lungo tutta la lunghezza del vaso riducendone il danno anatomico (5). La tecnica di puntura ad area definisce la concentrazione di ripetute punture in una zona limitata e circoscritta ed è la causa più frequente di sfiancamento aneurismatico del vaso o di retrazione stenotica subito a valle della puntura $(4,5)$.

Per questa ragione, essa deve essere limitata a situazioni "di 
emergenza", quando la presenza di particolari situazioni impedisce la scelta di altre tecniche (4).

La tecnica della puntura a occhiello (riservata alle FAV native) consiste nell'inserimento di un ago smusso sempre nel medesimo sito di incannulazione attraverso un tunnel cutaneo (occhiello) che viene formato mediante le prime venipunture effettuate dal medesimo operatore con aghi taglienti infissi con la stessa inclinazione (5).

Essa costituisce la vera alternativa alla puntura a scala di cor$\mathrm{da}$, in quanto riduce il dolore della venipuntura, riduce di gran lunga il margine degli insuccessi dovuto alla rotazione dei siti e non produce lo sfiancamento aneurismatico della parete del vaso (5-8).

Per questa ragione, i criteri raccomandati per la scelta dei pazienti da arruolare per la tecnica a sito costante (occhiello) sono:

- pazienti con FAV difficili o poco sviluppate

- pazienti con patrimonio vascolare ridotto

- pazienti con soglia del dolore elevata durante l'incannulazione

- autoincannulazione

- difficile emostasi

- estetica del braccio (9)

Il passaggio più delicato dell'applicazione della tecnica, che dura quattro settimane circa, è il periodo nel quale viene prodotto il tunnel dell'occhiello. Per affinare la tecnica di puntura a occhiello è indicato scegliere e coinvolgere all'inizio un numero limitato di pazienti. Dopo tale periodo, la metodica è strutturata e ci si può concentrare nuovamente su altri pazienti (4).

Questa tecnica richiede dei tempi di applicazione più lunghi rispetto a quella tradizionale anche quando l'occhiello è stato generato, in quanto necessita della rimozione delle croste della puntura precedente e di ripetute disinfezioni (10). Questa è un'altra ragione per la quale l'arruolamento dei pazienti deve essere limitato; infatti, si potrebbe correre il rischio di attuazione di "scorciatoie" per comprimere i tempi di applicazione. Diversi sono gli Autori che mettono in luce come, a fronte di benefici innegabili della tecnica (come, per esempio, la riduzione degli errori di incannulazione, la riduzione del dolore e la minore frequenza di stravasi ed ematomi), è necessario fare i conti con un aumentato rischio di infezione del sito di puntura $(11,12)$.

E innegabile che la presenza di una struttura anatomica (il tun$n e l$ ), che non c'è nella tecnica tradizionale, espone alla possibilità che in esso si trasportino e si annidino germi, per meccanismi simili a quelli che portano all'infezione del tunnel del catetere venoso centrale permanente. Da questo punto di vista, la tecnica va governata con l'applicazione rigida di protocolli di antisepsi condivisi dall' equipe infermieristica e dai pazienti arruolati, con la limitazione del numero dei pazienti arruolati a quel totale che il Centro Dialisi può gestire in assenza di rischi, con l'affinamento della tecnica e con la formazione adeguata del personale sull'argomento (12-17).

\section{Descrizione del problema e analisi del contesto}

Nell'Azienda USL di Rimini sono attivi tre Centri Dialisi: un Centro Ospedaliero (presso l'Ospedale di Rimini) e due
Centri di Assistenza Limitata (presso l'Ospedale di Riccione e l'Ospedale di Santarcangelo). Questi tre Centri forniscono un totale di 57 posti letto dialisi per pazienti con insufficienza renale cronica terminale attivi su 12 ore.

Ogni anno vengono confezionate, nella nostra Azienda, in media 46 nuove FAV; dal 2009 al 2013 la loro tipologia è completamente cambiata (Tab. I).

TABELLA I - INCIDENZA FISTOLE ARTERO VENOSE

\begin{tabular}{|l|c|c|c|c|}
\hline Incidenza accessi vascolari & FAV & FAV \\
distali & prossimali & $\begin{array}{c}\text { FAV } \\
\text { protesiche }\end{array}$ & $\begin{array}{c}\text { Nuove } \\
\text { FAV } \\
\text { totali }\end{array}$ \\
\hline 2009 & 30 & 18 & 7 & 55 \\
\hline 2010 & 20 & 26 & 9 & 55 \\
\hline 2011 & 16 & 15 & 4 & 35 \\
\hline 2012 & 10 & 29 & 3 & 42 \\
\hline 2013 & 12 & 26 & 4 & 42 \\
\hline
\end{tabular}

L'aumento della quota delle FAV prossimali porta facilmente a concludere come la popolazione incidente in dialisi giunge sempre più con un patrimonio venoso già scarso; ad essi si aggiungono i pazienti prevalenti che sono costretti al confezionamento di una nuova FAV per esaurimento della precedente. La fistola prossimale, per sua natura, presenta, in genere, un'estensione ridotta del tratto pungibile rispetto a quella distale (18). In questo contesto, la puntura a occhiello si inserisce come una buona opportunità per l'approccio alle FAV di difficile incannulazione e di scarsa estensione.

\section{Materiali e metodi}

\section{Popolazione}

L'oggetto di questo studio è rappresentato dai pazienti in emodialisi cronica arruolati per la puntura a occhiello dal 2009 al 2013 nei Centri Dialisi dell'Azienda Usl di Rimini.

\section{Obiettivi}

Riduzione del dolore da venipuntura

Risoluzione dei problemi di puntura nelle FAV "difficili”

Prevenzione delle infezioni locali del tunnel

\section{Protocollo utilizzato}

Per attuare la tecnica di puntura a occhiello, è stato utilizzato il Protocollo Specifico reso disponibile dal Presidente della filiale italiana EDTNA Marisa Pegoraro nella Pubblicazione "La puntura a occhiello della FAV arterovenosa" (5). Esso è stato implementato in due istruzioni operative di reparto prodotte nel 2009 e revisionate nel 2011.

La scelta dell'arruolamento viene fatta in modo multidisciplinare: medico nefrologo e infermiere del Centro Dialisi in accordo con il paziente.

Nei primi due anni, i criteri di inclusione/esclusione sono stati:

- l'intolleranza al dolore della venipuntura

- paziente giovane con FAV nativa nuova al fine di preservare l'aspetto estetico del braccio 
- FAV di estensione estremamente ridotta

Dal terzo anno, oltre a questi, sono stati introdotti i seguenti:

- assenza di terapia immunosoppressiva

- condizioni igieniche del paziente

- difficoltà di emostasi

\section{Strumenti}

È stato individuato un gruppo di pungitori che inizialmente era composto da tre infermieri a Rimini, due a Riccione e tre a Santarcangelo; a oggi i pungitori che hanno raccolto esperienza nella tecnica sono un gruppo composto da undici persone a Rimini e tre a Santarcangelo, mentre a Riccione tutto il gruppo è riuscito a sviluppare pari competenza; essi sono affiancati da un gruppo di miglioramento denominato "sorveglianza FAV" di cui fanno parte cinque infermieri a Rimini, due a Riccione, due a Santarcangelo e un medico nefrologo di riferimento sui tre Centri: questo gruppo collabora alla tenuta di dati relativi alle complicanze di tutte le FAV.

Per tutti i pazienti arruolati è stata compilata una scheda specifica che riportava, per ogni dialisi, tutte le informazioni necessarie. Per tre anni essa è rimasta in uso; poi, una volta consolidata la tecnica, è stata sostituita dalla scheda di sorveglianza FAV, comune a tutte le tipologie di fistole. Ad ogni paziente è stata fatta compilare la scheda della rilevazione del dolore con scala VAS almeno una volta all'anno. Nel 2013, è stata consegnata ai pazienti una scheda informativa compilata dal gruppo riguardo alla "manutenzione" dell'occhiello.

Ad ogni paziente è stata eseguita la prova del ricircolo almeno una volta all'anno; è stato calcolato il $\mathrm{kt} / \mathrm{v}$ due volte all'anno; ad ogni seduta sono stati registrati i parametri riguardanti la funzionalità della fistola stessa.

Ogni complicanza è stata registrata nella cartella infermieristica nella scheda di sorveglianza e, successivamente, i dati sono stati raccolti in un unico foglio di calcolo informatizzato. Ogni anno a tutti gli infermieri è stato somministrato un questionario anonimo per la valutazione della difficoltà percepita nella tecnica composto da poche domande a risposta chiusa (nelle quali è possibile anche più di una risposta) e da una parte a testo libero.

\section{Risultati}

Nella Tabella II sono riportati i dati di incidenza e prevalenza di ogni anno dei pazienti arruolati per l'occhiello del Centro Dialisi di Rimini.

TABELLA II - INCIDENZA E PREVALENZA BUTTONHOLE RIMINI

\begin{tabular}{|l|c|c|c|c|c|}
\hline CENTRO DIALISI RIMINI & $\mathbf{2 0 0 9}$ & $\mathbf{2 0 1 0}$ & $\mathbf{2 0 1 1}$ & $\mathbf{2 0 1 2}$ & $\mathbf{2 0 1 3}$ \\
\hline Arruolati Buttonhole & 18 & 10 & 0 & 4 & 4 \\
\hline Usciti dal Buttonhole & 0 & 3 & 12 & 4 & 4 \\
\hline Prevalenti Buttonhole al 31/12 & 18 & 25 & 13 & 13 & 13 \\
\hline
\end{tabular}

Nella Tabella III sono riportati i dati di incidenza e prevalenza di ogni anno dei pazienti arruolati per l'occhiello del Centro di Assistenza Limitata di Riccione.

TABELLA III - INCIDENZA E PREVALENZA BUTTONHOLE RICCIONE

\begin{tabular}{|l|c|c|c|c|c|}
\hline CAL RICCIONE & $\mathbf{2 0 0 9}$ & $\mathbf{2 0 1 0}$ & $\mathbf{2 0 1 1}$ & $\mathbf{2 0 1 2}$ & $\mathbf{2 0 1 3}$ \\
\hline Arruolati Buttonhole & 7 & 6 & 2 & 1 & 4 \\
\hline Usciti dal Buttonhole & 0 & 4 & 3 & 3 & 2 \\
\hline Prevalenti Buttonhole al 31/12 & 7 & 9 & 8 & 6 & 8 \\
\hline
\end{tabular}

Nella Tabella IV sono riportati i dati di incidenza e prevalenza di ogni anno dei pazienti arruolati per l'occhiello del Centro di Assistenza Limitata di Santarcangelo.

TABELLA IV - INCIDENZA E PREVALENZA BUTTONHOLE SANTARCANGELO

\begin{tabular}{|l|c|c|c|c|c|}
\hline CAL SANTARCANGELO & $\mathbf{2 0 0 9}$ & $\mathbf{2 0 1 0}$ & $\mathbf{2 0 1 1}$ & $\mathbf{2 0 1 2}$ & $\mathbf{2 0 1 3}$ \\
\hline Arruolati Buttonhole & 0 & 0 & 0 & 0 & 3 \\
\hline Usciti dal Buttonhole & 0 & 0 & 0 & 1 & 2 \\
\hline Prevalenti Buttonhole al 31/12 & 0 & 0 & 2 & 1 & 2 \\
\hline
\end{tabular}

Nella Tabella V, invece, si trovano i dati relativi alle infezioni locali verificatesi nei diversi anni, espresse in numeri assoluti.

TABELLA V - EPISODI INFEZIONE LOCALE BUTTONHOLE

\begin{tabular}{|l|c|c|c|}
\hline Infezioni locali Buttonhole & Rimini & Riccione & Santarcangelo \\
\hline 2009 & 4 & 0 & 0 \\
\hline 2010 & 8 & 4 & 0 \\
\hline 2011 & 2 & 0 & 0 \\
\hline 2012 & 1 & 2 & 0 \\
\hline 2013 & 1 & 2 & 0 \\
\hline
\end{tabular}

Per definire l'infezione si è fatto riferimento ai segni cardinali dell'infiammazione: rubor, tumor, calor, dolor, functio laesa. I pazienti sono stati sottoposti a terapia antibiotica per via orale o parenterale e i siti di puntura infetti sono stati immediatamente abbandonati.

Per quanto riguarda la valutazione della difficoltà da parte degli infermieri, i dati sono di difficile confronto di anno in anno, in quanto è diverso ogni volta il numero degli infermieri componenti l'equipe e perché ad ogni domanda può essere data più di una risposta:

- alla domanda "Quanto è difficile gestire la puntura a occhiello in una scala da 1 a 6 ?", la maggiore percentuale, stabilmente negli anni, si attesta sulla difficoltà 3 ;

- alla domanda "Quali sono state le maggiori difficoltà 
nell'applicazione della metodica che vorresti segnalare?", rimane stabile nel tempo una forte percentuale di difficoltà espressa nel mantenere l'angolo di inserzione durante l'incannulazione; al secondo posto, si attesta stabile nel tempo la difficoltà a rimuovere le croste. Inoltre, un'importante segnalazione è presente in merito alla difficoltà a condividere le scelte con i colleghi che non fanno parte del gruppo dei pungitori.

Infine, c'è la valutazione del dolore: tutti i pazienti arruolati hanno dichiarato una riduzione del dolore rispetto alla puntura tradizionale superiore al $60 \%$, tranne due pazienti, che ritengono che il dolore dell'effetto "elastico" e della rimozione delle croste sia superiore a quello dell'ago tagliente.

\section{Discussione}

Dai dati espressi nelle due Tabelle si può facilmente cogliere come l'entusiasmo iniziale abbia portato a un arruolamento troppo veloce di pazienti soprattutto nel Centro di Rimini; il salto compiuto per i pazienti prevalenti da 17 a 25 ha portato a veder raddoppiare gli episodi di infezione locale del tunnel. Successivamente, si assiste a un ridimensionamento del numero totale e, infine, al crollo delle complicanze infiammatorie.

Durante questo periodo sono state prodotte, inoltre, le due istruzioni operative sulla gestione della metodica, che sono state condivise con tutto il gruppo multidisciplinare.

E stata condotta, inoltre, una campagna di implementazione delle Linee Guida sull'igiene delle mani (19) ed è stata preparata e distribuita ai pazienti la scheda di "manutenzione" dell'occhiello.

Si evince, quindi, che l'affinamento della tecnica è andato di pari passo con diverse azioni compiute su più fronti, per ridurre il rischio di complicanze infettive a carico della FAV.

La criticità maggiore è stata quella di riuscire a gestire il pungitore unico in un Centro Dialisi che, fino al 2012, era aperto su tre turni, e la rotazione del personale sui diversi turni e sui diversi settori rendeva difficile la gestione organizzativa.

L'altra criticità è stata quella di vincere la resistenza degli infermieri stessi, che, alle prime complicanze, erano ben decisi ad abbandonare una tecnica che, comunque, richiede tempi più lunghi e maggiori attenzioni.

\section{Conclusioni}

La tecnica di puntura a occhiello piace alle persone in trattamento dialitico perché limita il dolore e l'ansia dell'incannu- lazione, mantiene una migliore estetica dell'arto e crea meno ematomi. È gradita anche allo staff infermieristico, almeno a una buona parte di esso. Sicuramente offre indiscussi vantaggi, ma presenta anche possibili complicanze che non devono essere trascurate. Una buona gestione degli aspetti igienici da parte del paziente e del personale è obbligatoria per ridurre il rischio infettivo.

\section{Riassunto}

La tecnica di puntura della fistola arterovenosa a occhiello consiste nel posizionare un ago smusso nella stessa sede. È una tecnica gradita ai pazienti perché riduce il dolore da venipuntura; è gradita anche al personale infermieristico, perché riduce i tempi di emostasi e minimizza la percentuale di insuccessi dell'incannulazione. Essa, però, richiede una rigida applicazione delle norme di buona pratica per la prevenzione delle infezioni in ambiente sanitario, in quanto presenta un possibile aumento dell'incidenza delle infezioni della cute.

Nei Centri Dialisi dell'Azienda USL di Rimini la tecnica è stata introdotta dal 2009 e, da allora, sono stati raccolti i risultati e i dati di sorveglianza delle complicanze infettive.

Parole chiave: Tecnica di puntura ad occhiello, Riduzione del dolore da venipuntura, Facilità di incannulazione, Infezione locale della cute

Dichiarazione di conflitto di interessi: Gli Autori dichiarano di non avere conflitto di interesse.

Contributi economici agli Autori: Gli Autori dichiarano di non aver ricevuto sponsorizzazioni economiche per la preparazione dell'articolo.

\section{Indirizzo degli Autori:}

Sgreccia Marina

Centro Dialisi di Rimini

Ospedale Infermi

Via Settembrini 2

47923 Rimini

marina.sgreccia@auslrn.net

\section{Bibliografia}

1. Libardi S, Bacchini G, Pontoriero G. La gestione dell'Accesso Vascolare nei pazienti in dialisi: il contributo dello Studio DOPPS. Giornale di Tecniche Nefrologiche \& Dialitiche 2010; XXII: 27-33. .

2. Tordoir J, Canaud B, Haage P, et al. EBPG on Vascular Access. Nephrol Dial Transplant 2007; 22 (Suppl. 2): ii88-117.
3. Quarello F, Forneris G, Pozzato M. La sorveglianza clinica e strumentale della fistola arterovenosa. G Ital Nefrol 2004; 21 : 317-30.

4. Segoloni G, Leonardi G (collaboratore). Gestione fistola artero-venosa per emodialisi; pubblicato il 15 Maggio 2013 sul portale Nephromeet consultabile all'indirizzo http://www. nephromeet.com/.

5. Pegoraro M. La puntura a occhiello della FAV arterovenosa. 
Storia e dati di evidenza nell'applicazione della tecnica. Giornale di Tecniche Nefrologiche \& Dialitiche 2008; XX: 1-6.

6. Verhallen AM, Kooistra MP, van Jaarsveld BC. Cannulating in haemodialysis: rope-ladder or buttonhole technique? Nephrol Dial Transplant 2007; 22 (9): 2601-4.

7. van Loon MM, Goovaerts T, Kessels AG, van der Sande FM, Tordoir JH. Buttonhole needling of haemodialysis arteriovenous fistulae results in less complications and interventions compared to the rope-ladder technique. Nephrol Dial Transplant 2010; 25 (1): 225-30.

8. Sukthinthai N, Sittipraneet A, Tummanittayangkoon B, Vasuvattakul S, Chanchairujira T. Buttonhole technique better than area puncture technique on hemostasis and pain associated with needle cannulation. J Med Assoc Thai 2012; 95 (Suppl. 2): S208-12.

9. Casarin R, Inf. Verdiglione G, Coord. Inf. Grilli R, Dr. Bonforte G. Puntura a occhiello o buttonhole. Tecnica di venipuntura della fistola arterovenosa (FAV). Esperienza centro dialisi Como, Azienda Ospedaliera S. Anna. Agorà n. 46, Dicembre 2010 consultabile sul sito: http://www.ipasvicomo.it/documenti/art_13_46.pdf.

10. Pegoraro M. Protocollo per la puntura a occhiello. Consultabile sul sito: http://www.ante.it/files/Seminari/Seminario\%20 Ante \%202007/PROTOCOLLO\%20PER\%20LA\%20PUNTURA\%20AD\%20OCCHIELLO.pdf

11. Atkar RK, MacRae JM. The buttonhole technique for fistula cannulation: pros and cons. Curr Opin Nephrol Hypertens
2013; 22 (6): 629-36.

12. Riva H, Dossi C, Bonforte G. Buttonhole: tutto il contrario di quello che ho sempre fatto. Giornale di Tecniche Nefrologiche \& Dialitiche 2013; 25: 89-91.

13. Labriola L, Crott R, Desmet C, André G, Jadoul M. Infectious complications following conversion to buttonhole cannulation of native arteriovenous fistulas: a quality improvement report. Am J Kidney Dis 2011; 57 (3): 442-8.

14. Birchenough E, Moore C, Stevens K, Stewart S. Buttonhole cannulation in adult patients on hemodialysis: an increased risk of infection? Nephrol Nurs J 2010; 37 (5): 491-8, 555.

15. Ball LK. The buttonhole technique: strategies to reduce infections. Nephrol Nurs J 2010; 37 (5): 473-7.

16. Van Eps CL, Jones M, Ng T, et al. The impact of extended-hours home hemodialysis and buttonhole cannulation technique on hospitalization rates for septic events related to dialysis access. Hemodial Int 2010; 14 (4): 451-63.

17. Doss S, Schiller B, Moran J. Buttonhole cannulation--an unexpected outcome. Nephrol Nurs J 2008; 35 (4): 417-9.

18. Berardinelli L, Frosini P, Gessaroli M. Proposta di Linee Guida per Accessi Vascolari per Emodialisi. Consultabile sul sito: http:// www.chirurgiavascolareroma.com/index 2.php?option=com docman\&task=doc_view\&gid=47\&Itemid $=18$.

19. Sgreccia M, Fronzoni T, Ticchi E, et al. La salute del paziente in dialisi e le nostre mani. Giornale di Tecniche Nefrologiche \& Dialitiche 2013: 25: 240-3. 